Prawo Kanoniczne

57 (2014) nr 1

ANDRIY TANASIYCHUK

Facoltà di Diritto Canonico S. Pio X, Venezia

\title{
ALCUNE QUESTIONI DI DIRITTO CONSACRATO NELLA LEGISLAZIONE CANONICA ORIENTALE
}

Sommario: - 1. Il valore e il luogo degli Istituti di vita consacrata nella Chiesa (cann. 410-411 CCEO). - 2. Il patrimonio dell'Istituto, cioè gli intendimenti e lo spirito del fondatore. (can. $426 \mathrm{CCEO}$ ). - 3. Classificazione (varietà) giuridica degli Istituti di vita consacrata. - 4. Il diritto proprio di ogni singolo Istituto. - 4.1. Il rapporto tra il CCEO e la legislazione propria. - 4.2. «Autonomia» degli Istituti di vita consacrata nei confronti dell'autorità ecclesiastica. - 4.3. «Direttorio» come raccolta delle risoluzioni e della decisioni delle Sinassi e anche come vademecum pratico. 4.4. Espressione «diritto particolare». - 5. Erezione o chiusura della casa monastica o religiosa. - 5.1. Comunità religiosa. - 5.2. Superiore. - 5.3 Apertura di una casa monastica. - 5.4. Chiusura di un monastero (=casa monastica). - 5.5. Apertura di una casa dell'Ordine della Congregazione o di una Società di vita comune a guisa dei religiosi. - 5.6. Soppressione della casa (cfr. can. 507; can. 510 CCEO). - Conclusione.

\section{Il valore e il luogo degli Istituti di vita consacrata nella Chiesa (cann. 410-411 CCEO) ${ }^{1}$}

San Paolo nella prima lettera ai Tessalonicesi ricorda che la vocazione principale di ciascuna comunità cristiana e di ogni suo singolo membro è la santificazione della vita (cfr. 1 Ts 4, 3). Essa si esprime tramite i diversi doni che attraverso l'opera dello Spirito Santo nascono nei fedeli. Questa santità si manifesta nelle varie forme della vita: nella vita laicale, nel sacerdozio e nella vita consacrata. Però si esprime in modo particolare tramite la pratica dei «consigli evangelici².

${ }^{1}$ Giovanni Paolo II, Codex Canonum Ecclesiarum Orientalium, 1990 (=CCEO), AAS 82, t. II (1990).

2 Cfr. Concilio Vaticano II, Cost. Dogm. Lumen Gentium, 39 (= LG). 
Alcuni fedeli ricevono la vocazione per vivere secondo questi «consigli evangelici»: «Lo stato costituito dalla professione dei consigli evangelici, pur non riguardando la struttura gerarchica della chiesa, appartiene tuttavia indiscutibilmente alla sua vita e alla sua santità $\rangle^{3}$. Questo stato di vita nell'insegnamento della Chiesa Cattolica viene chiamato lo stato consacrato: «I consigli evangelici della castità consacrata a Dio, della povertà e dell'obbedienza, fondati sulla parola e sull'esempio del Signore, e raccomandati dagli apostoli, dai padri e dai dottori e pastori della Chiesa, sono un dono divino che la chiesa ha ricevuto dal suo Signore e che con la sua grazia conserva sempre» ${ }^{4}$. Alcuni fedeli che sono stati santificati dal sacramento del Battesimo sotto l'opera dello Spirito Santo scelgono tale forma di vita, cioè vivere in una radicale consacrazione a Dio. Questa consacrazione, che trova le sue radici nella carità, che a sua volta si identifica con la natura di Dio, è indirizzata a costruire il Corpo Mistico di Cristo, che è la Chiesa creata per la salvezza del mondo. Inoltre tale consacrazione presenta un carattere escatologico, cioè la proclamazione del «Regno di Dio» ${ }^{5}$ e per questi motivi i fedeli devono favorire e sostenere questo stato di vita (cfr. can. 411 CCEO). Oltre a questo, la vita consacrata viene considerata come il segno della Chiesa e di una particolare vocazione divina. Paolo VI ricordava che le persone consacrate devono continuamente seguire Cristo perché questo è il segno di una risposta libera alla chiamata dello Spirito Santo ${ }^{6}$. Per questi motivi il fine e l'ispirazione di ciascun Istituto di vita consacrata è la loro attività missionaria per

3 Cfr. LG 44.

${ }^{4} \mathrm{LG} 43$.

${ }^{5}$ Cfr. CCEO, can. 410: «Lo stato religioso è un modo stabile di vivere in comune in un istituto approvato dalla Chiesa, nel quale i fedeli cristiani, seguendo più da vicino Cristo, Maestro ed Esempio di Santità, sotto l'azione dello Spirito Santo, con nuovo e speciale titolo sono consacrati per mezzo dei voti pubblici di obbedienza, castità e povertà da osservare sotto un legittimo Superiore a norma degli statuti, rinunciano al secolo e si dedicano totalmente a conseguire la perfezione della carità al servizio del Regno di Dio per l'edificazione della Chiesa e la salvezza del mondo, come segni che preannunciano la gloria celeste».

${ }^{6}$ Cfr. Paolo VI, Esortazione Apostolica Evangelica Testificatio, (29.06.1971), 7, EV 4, Bologna 1985, p. 639. 
la salvezza del mondo. Come fondamento teologico, per i primi due canoni generali del Titolo XII del Codice Orientale, viene considerato il magistero del Vaticano II. Il contributo teologico e disciplinare che esisteva fino ai tempi del Concilio, identificava la vita consacrata con lo stato perfetto, cioè lo stato che unisce le singole persone in una vita comune presso un Istituto di vita consacrata riconosciuto dalla Chiesa dove i membri godono di certi diritti e sono tenuti a determinati obblighi.

La vita consacrata è una scuola che vede il proprio fine nella perfezione e santità dell'ordinaria vita cristiana e anche diventano i testimoni della vita escatologica. D'altra parte questo stato di perfezione non si limita alla santità di vita solo del membro, ma deve essere indirizzata al servizio nella Chiesa. Perciò l'insegnamento dell'ultimo Concilio Ecumenico conferma: «Ma poiché i consigli evangelici in modo speciale congiungono coloro che li professano alla Chiesa e al suo mistero mediante la carità alla quale essi conducono, la loro vita spirituale deve perciò dedicarsi anche al bene della Chiesa intera. Ne deriva il dovere di lavorare per il regno di Cristo sia con la preghiera sia con l'operosa attività, ognuno secondo le proprie forze e secondo il genere della sua vocazione, perché il regno di Cristo si radichi e consolidi nelle anime, e si dilati in ogni parte della terra» ${ }^{7}$.

La vita consacrata, così come è stato detto in $L G$, si presenta come il segno, la rivelazione, la testimonianza e la proclamazione nell'intera Chiesa della presenza di Dio e la forza illimitata dello Spirito Santo. L'art. 44 include anche gli elementi di carattere disciplinare. Essi sono: erezione canonica delle singole forme di vita; la vita comune e stabilità dei membri che la hanno scelta; la vocazione per uno stile di vita escludendone automaticamente un altro; le professioni dei voti religiosi o le promesse dei consigli evangelici con qualche vincolo sacro; l'osservanza del diritto canonico e delle norme proprie dell' Istituto ${ }^{8}$.

Quando si parla di vita comune di un Istituto automaticamente vengono richieste due condizioni:

\footnotetext{
${ }^{7}$ LG 44.

${ }^{8}$ Cfr. D. Andrés, Le forme di vita consacrata, Roma 2005, p. 30.
} 
a). appartenere ad un gruppo di persone riunite in un certo Istituto ( $\mathrm{Mo}$ nastero, Ordine, Congregazione, Società di vita comune a guisa dei religiosi, Istituti secolari ed eretto canonicamente da parte della competente autorità ecclesiastica (Sede Apostolica, Patriarca, Vescovo eparchiale) (cfr. can. 414; can. 563 CCEO).

b). consacrazione della propria vita tramite $i$ voti e le professioni dei consigli evangelici con qualche vincolo sacro che chiaramente esprima i tre consigli evangelici: obbedienza, castità e povertà (cfr. can. 428; can. 462 §1; can. 531; can. 554 §1; can. 563 §1,1 CCEO).

\section{Il patrimonio dell'Istituto, cioè gli intendimenti e lo spirito del fondatore (can. $426 \mathrm{CCEO})$}

Tra le varie fonti del can. $426 \mathrm{CCEO}^{9}$ c'è il Decreto Conciliare del Vaticano II Perfectae Caritatis $^{10}$. Il Codice con precisione trasmette lo spirito di questo Documento sottolineando il grande valore che hanno gli intendimenti e le decisioni del fondatore: «Torna a vantaggio stesso della Chiesa che gli istituti abbiano una loro propria indole e una loro propria funzione. Perciò si riconoscano e si conservino fedelmente lo spirito e gli intendimenti propri dei fondatori, come pure le sane tradizioni: tutto ciò costituisce il patrimonio di ciascun istituto» ${ }^{11}$.

È chiaro che un Istituto di vita consacrata inizia la sua vita per volontà del fondatore stesso, che sotto ispirazione dello Spirito Santo arricchisce la missione della Chiesa. Inoltre occorre sottolineare che dopo la promulgazione di questo Documento, ciascun Istituto nella Chiesa Cattolica ha cominciato a percorrere una strada di studio e ricerca approfondita delle proprie radici per rivedere le intenzioni del proprio fondatore in maniera che potessero realizzarsi nelle situazioni contingenti ${ }^{12}$.

${ }^{9}$ Cfr. Pontificium Consilium de Legum Textibus Interpretandis, Codex Canonum Ecclesiarum Orientalium. Fontium annotatione auctus, Città del Vaticano 1995, can. 426, pp. 161-162.

${ }^{10}$ Cfr. Concilio Vaticano II, Decreto Perfectae caritatis $(=P C)$.

${ }^{11} \mathrm{PC} 2, \mathrm{~b}$.

${ }^{12}$ Durante il periodo 1991-2006, presso i diversi Istituti di vita consacrata che appartengono alla Chiesa Greco-Cattolica Ucraina, si sono svolti diverse Sinassi generali 
Il Codice orientale sottolinea un'altra particolarità, cioè rispetta con grande valore i principi di ciascun Istituto. Per questo motivo il compito principale di ogni Sinassi generale è la modalità di approfondire e rivedere il proprio «patrimonio» considerando e favorendo una duplice continuità: da una parte la fedeltà allo spirito principale e tradizionale dell'Istituto e da un'altra l'essere attenti alle necessità attuali della Chiesa secondo i propri compiti nei momenti dell'esercizio della propria missione nel mondo. Il Vaticano II esprime questo insegnamento nel modo seguente: «Il modo di vivere, di pregare e di agire deve convenientemente adattarsi alle odierne condizioni fisiche e psichiche dei membri, come pure, per quanto è richiesto dall'indole di ciascun istituto, alle necessità dell'apostolato, alle esigenze della cultura, alle circostanze sociali ed economiche; e ciò dappertutto, ma specialmente nei luoghi di missione» ${ }^{13}$.

\section{Classificazione (varietà) giuridica degli Istituti di vita consacrata}

Nella Chiesa Cattolica durante i secoli si è sviluppato il concetto di divisione tra i fedeli per ministri sacri e laici. Questa divisione trova i suoi inizi nei due sacramenti, cioè del battesimo e dell'ordine sacro.

e il loro frutto giuridico è stato per alcuni il cambiamento del proprio stato giuridico o, per altri, l'approvazione di nuovi Statuti. Così, per esempio, il 2 febbraio 1992 durante la Sinassi generale della Congregazione della Sacra Famiglia, è stata accettata la nuova versione dello Statuto insieme con le varie modifiche adattate ai tempi attuali. Questa Congregazione, fino al 1993 era nella condizione giuridica di diritto eparchiale. Dopo questa Sinassi è stato chiesto alla Sua Beatitudine Card. Myroslav Liubachivskiy, che in quei tempi era l'Arcivescovo-Maggiore della Chiesa Ucraina, di approvarli. Dunque il 21 ottobre 1993, a norma del diritto canonico orientale (cfr. 506 \$3 CCEO), questa Congregazione ha ottenuto riconoscimento come Congregazione di diritto patriarcale. In un altro caso nel luglio 2001, durante 8 Sinassi generali dell'Ordine delle Suore Basiliane (di diritto pontificio) furono introdotte innovazioni ed integrazioni alla loro legislazione che la Congregazione per le Chiese Orientali ha approvato il 26 marzo 2003.

Nell'anno 2005 è stata la Sinassi generale della Congregazione delle Suore Ancelle ( $d i$ diritto pontificio) che ha approvato a tempo determinato il nuovo Statuto che è entrato in vigore il 20 novembre 2006.

${ }^{13} \mathrm{Cfr}$. PC 3. 
Cosi come tramite recezione del battesimo tutti fedeli partecipano nel sacerdozio comune invece tramite la sacra ordinazione il fedele esercita il sacerdozio ministeriale. Inoltre ambedue Codici della Chiesa Cattolica sottolineano che questa diversità nel Popolo di Dio trova la sua fonte nel diritto divino (cfr. can. 207 \$1 CIC; can. 323 \$2 CCEO) ${ }^{14}$.

Oltre la divisione tra i fedeli per mezzo dei ricevuti sacramenti, la Chiesa conosce un alto tipo di classificazione del Popolo di Dio: chierici (cfr. can. 323 CCEO), laici (cfr. can. 399 CCEO) e consacrati (cfr. 410 CCEO). È necessario dire che la vita consacrata come un stato di persona diverso dagli altri stati seguito viene diviso in due forme: la vita contemplativa e la vita apostolica. Quindi le persone consacrate, anche se il loro stato si distingue dagli altri due stati di vita appartengono allo stesso modo alla vita e alla santità della Chiesa ${ }^{15}$.

Il Titolo XII del CCEO deve essere considerato come una revisione completa della legislazione precedente che trattava la vita consacrata nelle Chiese Orientali Cattoliche secondo il m. p. Postquam Apostolicis Litteris promulgata da Pio XII ${ }^{16}$. La legislazione attuale, così come la precedente, distingue tre principali Istituti di vita consacrata: Monasteri, Ordini, Congregazioni. Però in modo particolare si dà prevalenza ai Monasteri e alla vita monastica per evidenziare il vero spirito orientale che si esprime proprio nella vita monastica e che si distingue dagli Ordini e dalle Congregazioni ${ }^{17}$, anche se negli Ordini la professione perpetua è equiparata alla professione monastica (cfr. can. $533 \mathrm{CCEO}$ ), mentre nelle Congregazioni ha un valore giuridico minore (cfr. can. $534 \mathrm{CCEO})^{18}$.

${ }^{14}$ Cfr. Giovanni Paolo II, Codex Iuris Canonici, 1983, AAS LXXV (1983), Pars II.

${ }^{15}$ Cfr. D. Salachas, L. Sabbarese, Chierici e ministero sacro nel Codice latino e orientale, Città del Vaticano 2004, p. 35.

${ }^{16}$ Cfr. PIus XII, Motu Proprio Postquam Apostolicis Litteris, 9.02.1952, AAS 44 (1952), pp. 65-152.

${ }^{17}$ Cfr. Nuntia 16(1983), pp. 4-5.

${ }^{18}$ Cfr. Ibid., p. 6. È necessario fare un breve excursus storico circa il percorso della creazione degli Ordini religiosi nelle Chiese Orientali Cattoliche. Tale tipo di vita consacrata proviene dall'evoluzione del monastero tipico o, come viene chiamato nella scienza canonica, il monastero sui iuris in un Ordine religioso, con mescolanza degli elementi orientali di vita consacrata con gli elementi occidentali. Un esempio di questa 
È evidente che nelle Chiese Orientali Cattoliche le persone consacrate si chiamano con un termine comune: «monaci» e «monache», che sfortunatamente comprende anche le persone consacrate che appartengono ad un Ordine o ad una Congregazione. E' necessario mettere in luce che, da un punto di vista di diritto canonico e dal modo di vita, esiste una essenziale distinzione fra gli uni e gli altri. Il termine «vita monastica» corrisponde alle persone chiamate «monaci» $\mathrm{e}$ «monache ${ }^{19}$, che dimorano permanentemente in un Monastero sui iuris ${ }^{20}$ (si intende anche la casa monastica) o in un Monastero dipendente (filiale o sussidiario) ${ }^{21}$ per realizzare i fini monastici, conducendo una vita contemplativa ed esercitandosi nella propria santificazione personale. Essi non devono essere confusi con i semplici religiosi presso gli Ordini o le Congregazioni. Si deve ricordare che ogni monaco è un religioso, ma non ogni religioso è un monaco perché si distinguono nella propria organizzazione gerarchica, nella osservanza delle regole e nelle tradizioni della vita monastica (cfr. can. $433 \mathrm{CCEO})^{22}$. Le persone consacrate che appartengono ad un Ordine e ad una Congregazione vengono nominate religiosi.

trasformazione possono essere i monaci bizantini sotto la regola di S. Basilio Magno in Ucraina che nel sec. XVII, con la riforma del metropolita Rutski (1613-1637), si sono riuniti in una Congregazione della Santissimi Trinità (1617) e nell'anno 1631 hanno ottenuto la conferma da Roma. L'ultima riformulazione giuridica di tale ordine è stata fatta nell'anno 1954 anno in cui venne costituito l'Ordine religioso.

${ }^{19}$ Cfr. Concilio di Calcedonia, can. 4, in: Conciliorum Oecumenicorum Decreta, Bologna 1996, p. 89; Concilium Trullanum, can. 40, in: Fonti, fasc. IX, I, 1, Grottaferrata (Roma) 1962, p. 175-177. 187.

${ }^{20} \mathrm{Il}$ Monastero sui iuris è un monastero autonomo, cioè non subordinato ad un altro monastero e che nella propria vita monastica si regolarizza secondo il proprio Tipicon approvato dalla competente autorità ecclesiastica (cfr. can. 433 \$2 CCEO).

${ }^{21}$ I monasteri dipendenti possono essere di due tipi: filiali e sussidiari. «Quando si trova un monastero subordinato ad un altro, il più delle volte si tratterà d'un monastero o fondato per il servizio del principale, o che si è posto per motivi particolari sotto la dipendenza d'un altro monastero, in modo tale che il Superiore di questo monastero principale ha una vera autorità sul monastero subordinato, il quale a sua volta ha perduto la sua autonomia» - «Il monachesimo bizantino nella Legislazione del MP Postquam Apostolicis Litteris», in: Monachesimo Orientale, Orientalia Christiana Analecta 153, Roma 1958, p. 62.

${ }^{22}$ Cfr. C. Pujol, Il monachesimo bizantino ..., p. 77. 
Nel Titolo XII, che viene qui trattato, sono incluse anche le Società di vita comune a guisa dei religiosi, però senza i voti religiosi (cfr. can. 554 CCEO), nonché le Società di vita apostolica insieme con altre forme di vita consacrata (cfr. can. 570 CCEO) e quindi gli Istituti secolari (cfr. can. $563 \mathrm{CCEO}$ ). Si deve tener presente che i membri delle Società di vita comune a guisa dei religiosi non vengono chiamati religiosi, anche se hanno il modo di vivere dei religiosi. Negli Istituti secolari, invece, i membri possono essere sia dello stato religioso che laicale, ma non seguono la forma di vita dei religiosi (cfr. can. $563 \S 1,1$ CCEO).

È infine necessario ricordare che l'espressione «vita consacrata» nel CCEO chiaramente attribuisce ed indica tale tipo di vita soltanto per un Istituto di vita consacrata previsto dal Codice orientale.

\section{Il diritto proprio del singolo Istituto}

\subsection{Il rapporto tra il CCEO e la legislazione propria}

Nella legislazione comune per tutte le Chiese Orientali Cattoliche il Supremo Legislatore ha previsto la possibilità che gli Istituti di vita consacrata, nella propria vita interna, vengano guidati oltre che dal diritto comune anche da proprie norme e direttive, per sottolineare in questo modo una certa loro autonomia. Quindi quando si parla dei Monasteri, essi devono seguire le normative raccolte nel proprio Tipicon (cfr. can. $433 \$ 2$ CCEO) ${ }^{23}$, mentre per gli Ordini, le Congregazioni, le Società di vita comune a guisa dei religiosi e gli Istituti secolari sono previsti gli Statuti (cfr. can. 414 CCEO, can. 420 CCEO, can. 554 §1, can. $653 \S 1,2$ CCEO) e il diritto particolare delle Società di vita apostolica si chiama Costituzioni (cfr. can. 572 CCEO).

La collocazione canonica presentata sopra circa la legislazione propria degli Istituti non significa assolutamente che essi siano totalmente liberi nelle proprie decisioni nei confronti della vita intera per tutta la

${ }^{23}$ Questo principio è stato sottolineato anche dall'altro Pontefice in m. p. Ecclesiae sanctae di Paolo VI - cfr. Paolo VI, m. p. Ecclesiae sanctae, (6.08.1966) II, 16 §3, AAS 58(1966), p. 778. 
comunità o del singolo membro. Essi rimangono sotto la giurisdizione dell'autorità ecclesiastica che ha approvato o fondato l'Istituto stesso (cfr. can. 413 CCEO).

\section{2. «Autonomia» degli Istituti di vita consacrata nei confronti dell'autorità ecclesiastica}

Parlando dell'«autonomia» e dei suoi limiti nei confronti dell'autorità della Chiesa, che è rappresentata dal Gerarca del luogo e in modo particolare dal Vescovo eparchiale, è necessario constatare che essa si esprime per il fatto dell'esistenza della propria legislazione interna, che può essere modificata dallo stesso Istituto, però deve sempre essere approvata dall'autorità ecclesiastica alla quale è stato subordinato l'Istituto. Questo fatto è stato precisamente verbalizzato nei primi canoni di Titolo trattato. Così, per esempio, il can. 412 CCEO parla del voto di obbedienza verso il Romano Pontefice che può essere realizzato tramite una attenta lettura del Suo insegnamento e delle diverse «guide-istruzioni» prodotte dai vari dicasteri della Curia Romana, che successivamente trovano la loro applicazione nella vita concreta dei membri. Invece la materia che tratta la direzione e disciplina interna all'Istituto, è concessa dal Supremo Legislatore all'autorità ecclesiastica alla quale tale Istituto dirittamente appartiene (cfr. can. 413 $\mathrm{CCEO})^{24}$.

Parlando della competenza dell'autorità ecclesiastica nei confronti degli Istituti di vita consacrata è necessario constatare che il diritto comune per le Chiese Cattoliche Orientali precisa che esistono tre livelli di dipendenza giuridica (cfr. can. 414 §1, §2 CCEO) ${ }^{25}$ :

a). Istituti di diritto pontificio. Questi sono i Monasteri, gli Ordini, le Congregazioni, che sono stati fondati e riconosciuti da parte della Sede Apostolica con un decreto (cfr. can. 414 §2; can. $505 \S 1$, 2 (CEO) $)^{26}$;

${ }^{24}$ Cfr. S. Basilio Magno, Regular fusius tractatae, 35, in: Migne PG 31, p. 1003.

${ }^{25}$ Cfr. Pius XII, m. p. Postquam Apostolici Litteris, can. 24 §1, AAS 47(1952), pp. 73-74.

${ }^{26}$ Con il termine «Sede Apostolica» si intende la Congregazione per le Chiese 
b). Istituti di diritto patriarcale. La loro denominazione dipende dal loro stato canonico di dipendenza dal Patriarca/Arcivescovo-Maggiore. Questo stato canonico si può raggiungere con una duplice modalità: tramite la via ordinaria, cioè la fondazione o il riconoscimento con un decreto del Tipicon o degli Statuti (cfr. can. 414 §2; can. $505 \S 1, \S 2,2$; can. $506 \S 2$ CCEO) oppure tramite un decreto che cambia lo stato canonico dall'eparchiale al patriarcale (cfr. can. $506 \$ 3 \mathrm{CCEO})^{27}$;

c). Istituti di diritto eparchiale. Sono gli Istituti che in qualsiasi rapporto giuridico si trovano sotto la giurisdizione del Vescovo eparchiale. È necessario ricordare che la legislazione orientale distingue i tre tipi principali di Istituti di vita consacrata, ma il Vescovo eparchiale può erigere soltanto i Monasteri e le Congregazioni (cfr. can. 414 $\S 1,1$; can. 435 \$1 CCEO) mentre la potestà di fondare gli Ordini non gli è stata data da parte del Supremo Legislatore (cfr. can. 505 $\S 1$ CCEO).

In particolare, il Vescovo eparchiale, sul territorio dove egli esercita il governo come Gerarca del luogo, prima di emanare il decreto della creazione della prima casa (principale) di tale Istituto, deve chiedere il parere sia alla Sede Apostolica, sia al Patriarca/Arcivescovo Maggiore (cfr. can. $414 \S 1$; can. $506 \S 1$ CCEO). Invece, se il Monastero o la Congregazione si diffonde in altre eparchie ed esarcati o se si tratta di introdurre qualche cambiamento nei propri Tipicon o Statuti, tale Vescovo eparchiale, dove si trova la casa principale, prima di confermare le innovazioni, per la validità del proprio atto amministrativo, deve sentire il consiglio degli altri Vescovi dei territori in cui si trovano le altre case (cfr. can. $414 \S 3$ CCEO).

Orientali - cfr. Giovanni Paolo II, Cost. Ap. Pastor Bonus (28.06.1988), 58.

${ }^{27}$ Dopo il trasferimento della sede dell'Arcivescovo-Maggiore a Kiev (dicembre 2004), questa Congregazione è rimasta l'unica Congregazione di diritto patriarcale mentre prima, quando la sede dell'Arcivescovo-Maggiore si trovava in Leopoli (Lviv), sotto la sua giurisdizione si trovavano anche le altre Congregazione però solamente di diritto eparchiale in quanto egli era il Vescovo eparchiale (cfr. can. 101; can. $414 \S 1$, $\S 2 \mathrm{CCEO})$. 
In passato la presunta «autonomia» era segnata dalla continua ingerenza nella vita dell'Istituto da parte dell'autorità ecclesiastica ${ }^{28}$. Diversamente, il diritto canonico attuale (CCEO), ha voluto sottolineare l'aspetto della reciprocità fra l'autorità ecclesiastica e gli Istituti di vita consacrata anche di diritto pontificio. Queste relazioni reciproche si evidenziano espressamente nel momento del riconoscimento del Tipicon, degli Statuti o delle Costituzioni (cfr. can. 414 CCEO), quando si concede il decreto di esclaustrazione (cfr. can. 489; can. 548 CCEO) o quando si tratta di attività apostolica nell'eparchia (cfr. can. $415 \S 1$, $\$ 2$ CCEO). Per quanto riguarda la vita interna dell'Istituto, invece, il Supremo Legislatore mette qualche limite d'intervento per l'autorità ecclesiastica a meno che non siano sorti degli abusi in una singola casa o il delitto sia stato compiuto da un membro della casa religiosa ma fuori dalla stessa casa (cfr. can. 417 CCEO).

Il Supremo Legislatore concede al Vescovo eparchiale il diritto, che è posto anche come obbligo, di visitare le singole case dei Monasteri o degli Ordini e le Congregazioni, che sono state fondate da lui stesso o che si trovano nel territorio della sua eparchia/esarcato. Il Vescovo eparchiale deve visitare anche gli altri Istituti di vita consacrata che sono coinvolti nell'apostolato eparchiale o quando lo consigliano cause gravi (cfr. can. 414 §1,3; 415 § CCEO).

Dall'esperienza quotidiana delle diverse eparchie o esarcati nei territori delle quali si trovano diverse case religiose o monasteri, il Vescovo eparchiale può chiedere che il religioso compia qualche lavoro nell'evangelizzazione. Questo conferimento del compito deve essere preceduto dal permesso del Superiore competente del religioso ${ }^{29}$. Sa-

${ }^{28}$ Cfr. Concilio di Calcedonia, can. 4; Concilium Trullanum, can. 41, can. 49, in: Fonti, fasc. IX, I, 1, cit., pp. 177-179. 187; I-II Concilio di Costantinopoli (861), can. 1, can. 2; Corpus Iuris Civili, Novellae, CXX, VI; CXXXIII, IV, Hildesheim 1993, pp.582-585. 671-672.

${ }^{29}$ CCEO, can. 418 §1: «Sono Superiori maggiori il Preside di una confederazione monastica, il Superiore di un monastero sui iuris, il Superiore generale di un ordine o di una congregazione, il Superiore provinciale, i vicari degli stessi e gli altri che hanno una potestà a guisa dei Provinciali, come pure coloro che, quando mancano i predetti, succedono legittimamente nell'ufficio. 
rebbe utile, a norma del can. $282 \S 2$ CCEO, che fosse stipulato, fra il Vescovo eparchiale e quello Superiore, un contratto circa il servizio del religioso nella eparchia/esarcato. In tale contratto si devono esporre in modo chiaro i diritti e gli obblighi di questo membro.

Ove si parla dell'apostolato in generale, il Supremo Legislatore pone anche certi limiti per i membri. Per esempio, quando si tratta del tempo di noviziato presso i Monasteri (cfr. $459 \S 2$ CCEO) o presso gli Ordini e le Congregazioni (cfr. can. $523 \S 1$ CCEO); o quando si tratta di nominare un monaco come parroco o di creare una parrocchia presso il Monastero. In questi ultimi casi, se si tratta di aspetti relativi all'interno del territorio patriarcale, occorre ricevere il permesso dal Patriarca, invece in tutti gli altri casi dalla Sede Apostolica (cfr. 480 CCEO). Quando si tratta dei diritti del Patriarca verso i monaci di un Monastero o i religiosi di un Ordine o di una Congregazione, lo stesso Patriarca può incaricare uno di essi per il compimento di qualche incarico relativo all'intera Chiesa sui iuris, però prima deve consultare il Superiore maggiore o, nel caso in cui sia previsto dal diritto particolare della Chiesa sui iuris, deve avere anche il suo consenso (cfr. can. 89 $\$ 2 \mathrm{CCEO})$.

Occorre ricordare che il conferimento di tale compito non dispensa il membro dalle norme del proprio diritto particolare o dalla disciplina monastica o religiosa. Esercitando il dato incarico il membro deve conservare il carattere e la specificità del proprio Istituto (cfr. can. $415 \S 3$; can. 543 CCEO). Anche nel foro giudiziario, se il Superiore proprio non punisce il membro colpevole, questo compito passa al Gerarca del luogo (cfr. can. 415 \$4 CCEO) o al Patriarca (cfr. can. 1061; can. $1063 \S 4,3-4$ CCEO). Invece quando si tratta di abusi di vario tipo in una casa e il Superiore di essa non reagisce, il Gerarca del luogo è obbligato a trasmetterne notizia all'autorità ecclesiastica che ha eretto o riconosciuto tale Istituto (cfr. can. $417 \mathrm{CCEO}$ ).

§2. Sotto il nome di Superiore di monaci e di tutti gli altri religiosi non è compreso il Gerarca del luogo né il Patriarca, fermi restando i canoni che attribuiscono al Patriarca o al Gerarca del luogo una potestà su di loro». 
4.3. «Direttorio» come raccolta delle risoluzioni e delle decisioni delle Sinassi e anche come vademecum pratico

Il can. $441 \S 1$ e il can. $511 \S 1$ CCEO quando parlano circa l'esistenza e il compito delle Sinassi generali esprimono la volontà del Supremo Legislatore che vuole sottolineare il grande valore giuridico delle risoluzioni e delle decisioni che in esse vengono prese. Queste prescrizioni e disposizioni generali, creano una raccolta di leggi che si distingue da quella principale ${ }^{30}$ ed ha il compito di regolare la vita interna dell'Istituto adattando le norme principali alle occorrenze attuali. Questo significa che tutti gli Istituti oltre al diritto particolare nella forma sia del Tipicon che degli Statuti hanno anche altre norme giuridiche $^{31}$ su cui fare riferimento. Quindi è opportuno, per motivi pratici, che tutte le risoluzioni e le decisioni siano raccolte in un volume chiamato «Direttorio». La particolarità di tale raccolta si trova nell'applicazione e adattamento pratico del Tipicon o degli Statuti ai tempi e alle richieste del luogo per tutta la provincia o per una singola casa.

Può sorgere la domanda: come deve essere il rapporto tra queste due normative (Tipicon/Statuti e Direttorio)?; quanti possono essere questi Direttori: possono essere raccolti in un unico volume o in vari volumi?

Il motivo della quantità di queste raccolte può dipendere per esempio dalla varietà dell'apostolato nelle varie province che nello stesso tempo si trovano in diversi Stati ${ }^{32}$. Esistono diverse possibilità per regolare il rapporto tra le norme principali e secondarie e per scegliere la forma che diventi più efficace al raggiungimento del fine preposto. Per esempio si può:

${ }^{30}$ Il Tipicon è proprio dei Monasteri di qualsiasi condizione giuridica, gli Statuti sono propri degli Ordini, delle Congregazioni e delle Società di vita comune a guisa dei religiosi.

${ }^{31}$ Questa norma è prevista espressamente solo nel can. 587 §4 CIC.

${ }^{32}$ Cfr. La Costituzione delle Suore Ancelle dell'Immacolata Concezione, art. 63, Roma-Lviv 2006, p. 3. Questa Congregazione è di diritto pontificio ed appartiene alla Chiesa Greco-Cattolica Ucraina. La sua legislazione è stata nominata come la «Costituzione» e non come lo «Statuto». 
a). fare la redazione del Tipicon/Statuti e del «Direttorio» come due libri diversi;

b). pubblicarli in un unico libro, però uno dopo l'altro indicando: I parte - Tipicon/Statuti e II parte - «Direttorio»;

c). collocarli in un unico libro, senza distinguere tra I e II parte, ma mettendo prima il capitolo del Tipicon/Statuti e dopo il capitolo del «Direttorio».

Il contenuto del «Direttorio» deve includere le norme e le decisioni del Capitoli oltre a:

a) le decisioni fatte secondo lo spirito e la necessità contingente e la loro l'applicazione pratica nella vita reale;

b) quello che bisogna fare secondo le necessità e le circostanze attuali;

c) quello che è secondario e non coincide con la natura dell'Istituto;

d) le norme pratiche che ogni istituzione deve osservare per il proprio bene e per essere più efficaci.

$\mathrm{Ci}$ si può chiedere se questo significherebbe che tale «Direttorio» avrebbe soltanto un carattere giuridico - pastorale senza nessun riferimento alla vita spirituale o morale? Negativo! Questo «Direttorio» oltre che esprimere il carattere giuridico - pastorale potrebbe diventare anche una giuda spirituale di ciascun Istituto di vita consacrata.

\subsection{Espressione «diritto particolare»}

Il diritto comune delle Chiese Orientali Cattoliche nel Titolo XII in alcuni canoni usa il termine «diritto particolare»" Nell'interpretazione di questa espressione occorre essere molto attenti perché questo termine non sempre vuole intendere il diritto particolare di una Chiesa sui iuris o il diritto eparchiale, ma comprende, oltre al Tipicon/Statuti, anche l'altra legislazione interna dei Monasteri, degli Ordini e delle Congregazioni (decisioni, decreti, rescritti). Così, per esempio, il can. $422 \$ 2$ CCEO dove viene menzionato questo termine, dice che: «nel diritto particolare si stabilisca se nelle case dove vivono meno di sei membri, il consiglio debba esserci o no». Nella redazione di questo

\footnotetext{
${ }^{33}$ Cfr. can. 422 §2; can. 496 §2; can. 499; can. 554 §2; can. 569; can. 570; can. 572 CCEO.
} 
canone è stato utilizzato il termine «diritto particolare» che non intende il diritto particolare di una Chiesa sui iuris, ma un regolamento interno di una singola casa religiosa. Perciò ogni Monastero, Ordine e Congregazione nella propria legislazione (Tipicon/Statuti, «Direttorio») deve provvedere alla costituzione di una norma che obblighi ogni casa religiosa ad avere un regolamento o, nelle case dove ci sono meno di sei membri e se la situazione lo richiede, che ci sia un consiglio della casa stessa $^{34}$.

È permesso ad un Monastero, ad un Ordine e ad una Congregazione di avere, oltre alla propria legislazione, anche delle altre norme particolari stabilite per la singola provincia? È necessario dire di si, però sotto condizione, se sono state previste dalle norme principali dell' Istituto, e devono conservare l'unità e raggiungere lo scopo dell'Istituto. Esse devono prevedere:

a). in quali casi la singola provincia può avere delle norme proprie;

b). chi è competente a promulgare tali norme (nella provincia).

Queste norme particolari non devono essere emanate senza le necessità previsti e possono regolarizzare la vita pratica della provincia e i suoi aspetti giuridici.

\section{Erezione o chiusura della casa monastica o religiosa}

Per quanto riguarda l'erezione o la chiusura della casa di qualsiasi Istituto di vita consacrata è necessario essere consapevoli del valore di tale casa per la Chiesa Cattolica in generale e in modo particolare per una singola eparchia/esarcato. Queste case sono un luogo giusto per la santificazione della propria vita e il loro compito principale si esprime nel dare il buon esempio di come si compie tale dovere. La vita consacrata include la rinuncia alla vita secolare e al suo modo di agire e porta ad accettare un altro stile di vita, che è tipico per ogni singolo Istituto: la preghiera, l'osservanza delle regole, il silenzio, la solitudi-

${ }^{34}$ Cfr. Nuntia 16(1983) p. 19. 
$\mathrm{ne}^{35}$. I diversi canoni del Titolo XII presentano le norme che cercano di regolarizzare tale vita monastica o religiosa ${ }^{36}$.

\subsection{Comunità religiosa}

Con il termine comunità religiosa si considera il gruppo di persone che creano una comunità. Invece con l'espressione la casa, usato dal Legislatore, non si considera soltanto una struttura architettonica indipendente, ma anche un semplice appartamento. È chiaro che l'elemento principale della comunità religiosa è la comune convivenza dei membri sotto uno stesso tetto. Non si può dire che esista una comunità senza l'espressione della vita comune (cfr. can. 478; can. 495; can. 550 CCEO). Il Legislatore non proibisce ai membri di un Istituto di vivere fuori dalla casa religiosa però questa situazione richiede una valida giustificazione (cfr. can. $478 \mathrm{CCEO}$ ). Non è possibile chiamare un gruppo di persone come comunità se esse si incontrano tra di loro solo ogni tanto.

\subsection{Superiore}

Ogni comunità religiosa deve avere un superiore che viene eletto secondo le norme di diritto canonico e della propria legislazione particolare. Per esempio quando si parla del superiore nei Monasteri sui iuris occorre sottolineare che egli viene eletto durante le Sinassi generali presiedute dal Vescovo eparchiale ${ }^{37}$. Inoltre 1'eletto superiore può svolgere due ruoli: come Superiore generale e come superiore della casa monastica (cfr. can. $443 \S 1 \mathrm{CCEO})^{38}$. La durata dell'esercitazione

\footnotetext{
${ }^{35}$ Cfr. J. Beyer, Il diritto della vita consacrata, Milano 1989, p. 209.

${ }^{36}$ Cfr. can. 437 §2; §3; can. 438; can. 509; can. 510; can. 556 CCEO.

${ }^{37}$ Nelle Chiese Orientali Cattoliche questo diritto del Vescovo eparchiale di presiedere durante le Sinassi generali di un Monastero sui iuris esisteva già dall'antichità - cfr. Synodus Zamosten. Ruthenorum, a 1720,tit. XII, "Electiones" in: Mansi XXXV, p. 1518; Syn. LibANEN MARONITARUM, a 1736, pars IV, cap. III, 11, in : Mansi XXXVIII, p. 248; Syn. Leopolien. Ruthenorum, a 1891, tit. X, 5, in: Acta et Decreta Sinodi Provincialis Ruthenorum habita in Leopoli, anno 1891, Roma 1896, p. 219.

${ }^{38}$ In Ucraina in ciascun monastero dipendente (filiali o sussidiari), di regola di san Teodoro Studita, esiste un superiore; invece il Superiore generale di tutto il Monastero
} 
di questi uffici dipende da quanto stabilito nel Tipicon (cfr. can. $444 \S 1$ $\mathrm{CCEO}{ }^{39}$. Invece nei Monasteri dipendenti, filiali e sussidiari (cfr. can. $436 \S 1$ CCEO), la figura del superiore viene nominata dal Superiore del monastero sui iuris e include soltanto il governo interno di una casa monastica e viene sempre eletto a tempo determinato (cfr. can. $444 \S 2$ CCEO).

Presso gli Ordini, le Congregazioni e le Società di vita comune a guisa dei religiosi esiste un procedimento canonico diverso per diventare o il Superiore generale, o il superiore di una casa. Se si tratta di Superiore generale di un Ordine o Congregazione egli viene designato mediante elezioni canoniche fatte a norma del diritto canonico e dei propri Statuti (cfr. can. $515 \S 1$ CCEO). Se si tratta di superiore di una singola casa religiosa di questi Istituti può essere sia eletto secondo gli Statuti, e in questo caso occorre ottenere l'approvazione dal Superiore maggiore che può essere il provinciale (cfr. can. 418 §1 CCEO), che nominato dallo stesso Superiore maggiore (cfr. can. 515 \$2 CCEO). La stessa procedura viene osservata quando si deve eleggere un Superiore maggiore di una Società di vita comune a guisa dei religiosi.

È chiaro che l'elemento principale di ogni casa monastica e religiosa è il luogo dedicato alla preghiera, cioè la cappella. Se si tratta di un Istituto clericale ${ }^{40} \mathrm{il} \mathrm{monastero} \mathrm{potrebbe} \mathrm{essere} \mathrm{anche} \mathrm{la} \mathrm{propria} \mathrm{chiesa}$ nella quale viene celebrato e conservato il Santissimo Sacramento ${ }^{41}$.

\subsection{Apertura di una casa monastica}

La singola comunità di ciascun Istituto ha il pieno diritto di abitare in una casa canonicamente fondata. Però per capire meglio le norme canoniche che riguardano la sua fondazione o la sua chiusura, è necessario introdurre alcune spiegazioni circa la terminologia usata dal

sui iuris, al quale appartengono quelli dipendenti, si chiama Igumeno - cfr. Tipicon Comune dei Monasteri Studiti, Lviv 1996, p. 75 [Zagalnyj Typikon Studytskykh Monastyriv, Lviv 1996, 75]

${ }^{39}$ Cfr. Tipicon Comune dei Monasteri Studiti, Leopoli 1996.

${ }^{40} \mathrm{Si}$ intende sia Ordine che Congregazione (cfr. can. 509 \$3 CCEO).

${ }^{41}$ Cfr. Can. 282; can. $284 \S 1$; can. 415 §1; can. $437 \S 1$; can. 480 CCEO. 
Codice. Il termine «monastero» è usato dal Legislatore oltre che per indicare un Istituto di vita consacrata, anche per indicare la casa dove abitano i monaci o le monache. Quindi quando si parla di un Monastero si intende, oltre che una persona giuridica nella Chiesa, anche la semplice casa monastica.

Quando si parla dell'apertura di una casa di qualche Istituto di vita consacrata è necessario distinguere quale è il tipo di Istituto (Monastero sui iuris o monastero dipendente, Ordine, Congregazione, Società di vita comune a guisa dei religiosi) che vuole fondare una nuova casa. Occorre sottolineare anche il fatto che il Supremo Legislatore ha previsto diverse forme canoniche nella procedura per aprire una casa. Tale processo dipende dal tipo di Istituto che intende fare questo. Così, per esempio, per aprire un monastero dipendente, filiale e sussidiario, (=casa monastica), il Monastero sui iuris deve ottenere il permesso scritto sia dell'autorità ecclesiastica alla quale è subordinato che del Vescovo eparchiale del luogo dove si intende fondare un nuovo monastero (=casa monastica) (cfr. can. $436 \$ 2$ CCEO). Invece se il Monastero sui iuris o un monastero dipendente esistente in una eparchia/ esarcato, desiderasse aprire una casa, simile ad un ospizio, separata da essi, prima di compiere tale atto giuridico devono ricevere nuovamente il permesso dal Vescovo eparchiale del territorio su quale si trovano (cfr. can. 437 § $\mathrm{CCEO}$ ).

\subsection{Chiusura di un monastero (=casa monastica)}

Quando si tratta della chiusura del Monastero nel senso di una casa monastica è necessario distinguere: 1) di quale Monastero si tratta: $d i$ un Monastero sui iuris, un Monastero dipendente di diritto eparchiale, un monastero stauropegiaco ${ }^{42}$, la confederazione dei monasteri sui

${ }^{42}$ Il titolo stauropegiaco “(...) si riferisce all'antico rito della dedizione e benedizione di un monastero, in cui si fissava una croce sul luogo del futuro altare. Il termine più tardi venne riservato ai monasteri sottoposti al diretto controllo del Patriarca" - D. SALaCHAs, Il Diritto Canonico delle Chiese orientali nel primo millennio, Bologna 1997, p. 172. Inoltre nel 1983 il Gruppo di studio della Pontificia Commissio Codici Iuris Canonici Orientalis Recognoscendo nella nuova revisione dei canoni «De Monachis ceterisque Religiosis necnon de sodalibus aliorum Institutorum vitae consecratae», vo- 
iuris $^{43}$; 2) su quale territorio si trova, cioè se dentro al territorio della Chiesa patriarcale o fuori ${ }^{44} ; 3$ ) quale autorità ecclesiastica è competente ad emanare il decreto di chiusura. Per questo l'elemento principale che viene in grande considerazione è il territorio dove esso è stato costituito.

Quindi quando si tratta di un Monastero sui iuris o di un Monastero filiale di diritto eparchiale e di un Monastero stauropegiaco, che si trovano dentro del territorio della Chiesa Patriarcale, tale competenza appartiene soltanto al Patriarca. Però esistono due procedimenti diversi per emanare il decreto di soppressione. Quando si tratta di Monasteri stauropegiaci per la validità del decreto si deve ottenere il consenso del Sinodo Permanente e costatare se esistono gravi cause. Inoltre se questi Monasteri sono di diritto eparchiale e l'iniziativa di soppressione proviene dal Vescovo eparchiale si richiede che il Patriarca riceva per iscritto tale richiesta dal Vescovo eparchiale o almeno che lo consulti (cfr. can. $438 \S 1$; can. 934 §2 CCEO).

Invece quando questi Monasteri sono riuniti in una confederazione e si trovano dentro al territorio della Chiesa Patriarcale, il Patriarca prima di emanare il decreto di soppressione deve sentire il parere del Superiore del monastero o il Preside della confederazione e ottenere il consenso del Sinodo Permanente (cfr. can. 438 §1 CCEO).

leva precisare e nello stesso tempo evitare per il futuro ogni dubbio circa la posizione giuridica del Monastero stauropegiaco. Questo tipo di Monastero può essere paragonato all'Abbazia territoriale nella Chiesa latina (cfr. can. $370 \mathrm{CIC}$ ), però con una, o direi, essenziale differenza che il Gerarca del luogo non è Igumeno (=Abbate), ma il Patriarca stesso. Questo riferimento al Patriarca significa che il Monastero stauropegiaco è esente dalla potestà del Vescovo eparchiale del territorio sul quale esso si trova e dipende giuridicamente dal Patriarca (cfr. can. 435 \$2 CCEO).- cfr. can. 263 m.p. Cleri sanctitati, AAS 49(1957), p. 513; Nuntia 16(1983), p. 60.

${ }^{43}$ Questo tipo di vita dei Monasteri sui iuris in una confederazione, fu prevista anche nella legislazione precedente, cioè nel m. p. Postquam Apostolicis Litteris (9.02.1952) di Pio XII. Inoltre è necessario sottolineare che già in passato esisteva la pratica di riunire singoli monasteri in una confederazione - cfr. Synodus Zamosten. RUtHENORUM, a 1720, tit. XI "Cum experientia" in: Mansi XXXV, p. 1515; Syn. Libanen MaronitaRUM, a 1736, pars IV, cap. III, 2, in : Mansi XXXVIII, p. 247.

${ }^{44}$ Competenza della Congregazione per le Chiese Orientali - cfr. Pastor Bonus, 58. 
Quando si tratta della chiusura di un monastero sussidiario, che è diverso dal monastero filiale, può emanare il decreto di soppressione il Superiore del monastero sui iuris, da cui dipende quel monastero e dopo aver ricevuto il nulla osta dal Vescovo eparchiale, (cfr. can. 438 $\$ 3 \mathrm{CCEO}$ ). In altri casi, cioè quando i Monasteri di diritto eparchiale o pontificio si trovano nel territorio di una Chiesa sui iuris sprovvisto della propria gerarchia ecclesiastica, tale competenza spetta alla Sede Apostolica (cfr. can. $438 \S 2$ CCEO) $)^{45}$.

5.5. Apertura di una casa dell'Ordine, della Congregazione o di una Società di vita comune a guisa dei religiosi

Parlando della procedura canonica che deve essere osservata nel procedimento di apertura di una casa da parte dell'Ordine, della Congregazione o della Società di vita comune a guisa dei religiosi, occorre segnalare che il Codice orientale distingue due tipi di situazioni: l'apertura di una nuova casa nel territorio della stessa Chiesa sui iuris, dove già esistono altre case dello stesso Istituto o l'apertura di una prima casa nel nuovo territorio che è anche sprovvisto della propria gerarchia ecclesiastica. Questa distinzione porta anche una diversità di requisiti canonici che devono essere osservati nel processo di legittima apertura della casa (cfr. can. 509 CCEO).

Quando si tratta di aprire una nuova casa sono necessari:

- la decisione dell 'Ordine, della Congregazione, della Società di vita comune a guisa dei religiosi. Per considerare la legittima apertura di una nuova casa dell'Istituto è necessario che essa sia eretta dalla

${ }^{45}$ Qui non viene detto nulla circa la chiusura del Monastero stauropegiaco, perché quello non può esistere in un territorio canonico di un'altra Chiesa sui iuris dove il patriarca non ha giurisdizione. È necessario ricordare che solo i monasteri di diritto eparchiale e pontificio possono essere aperti in un nuovo territorio dove non esiste la propria gerarchia ecclesiastica. Essi vengono sottomessi alla giurisdizione del Vescovo eparchiale/diocesano (cfr. can. $414 \S 1,3$; can. $415 \S 1$, 2 CCEO). Invece il monastero stauropegiaco, essendo sotto la potestà del Patriarca, dove egli svolge il governo come il Vescovo eparchiale (cfr. 101 CCEO), non può esistere fuori del territorio della Chiesa patriarcale o arcivescovile-maggiore, perché la giurisdizione del Patriarca è limitata nel territorio dove egli presiede (cfr. can. 78 \$2 CCEO). 
competente autorità di questo Istituto che deve seguire le norme del proprio diritto particolare (Tipicon, Statuto, Costituzione). Per questo motivo si raccomanda che nel diritto proprio di ciascun Istituto venga chiaramente indicata l'autorità dentro l'Istituto (superiore generale, superiore provinciale, sinassi generale o provinciale) che sia competente ad erigere una nuova casa ${ }^{46}$;

- il permesso scritto del Vescovo eparchiale e non soltanto una semplice benedizione ${ }^{47}$. Il Supremo Legislatore richiede, e non soltanto raccomanda, che l'atto giuridico dell'Istituto sia accompagnato con la lettera del Vescovo eparchiale che permette tale passo giuridico. Questo requisito non è facoltativo, ma è obbligatorio, e viene richiesto come un elemento sostanziale per la validità dell'atto amministrativo. Perciò soltanto dopo aver ricevuto tale permesso si può procedere alla sistemazione delle formalità e del processo. Occorre sottolineare che il diritto canonico usa il termine "Vescovo eparchiale», che ontologicamente si distingue dal «Gerarca del luogo» (cfr. can. 984 § CCEO). Questo significa che il Protosincello (=Vicario generale nel CIC), che può essere anche il Vescovo ausiliare, non è competente a dare tale documento.

Inoltre è necessario sottolineare che questo permesso, richiesto nell'ambito giuridico da parte del Vescovo eparchiale, ha un concetto largo e non è soltanto come un requisito della norma canonica. Con esso i membri della casa ricevono certi diritti previsti dalla norma canonica: vivere secondo il carattere e i fini dell'Istituto; esercitare i doveri previsti dall'Istituto secondo le norme di diritto canonico, della propria legislazione e secondo le disposizioni date dal Vescovo eparchiale se sono coinvolte nell'apostolato. Invece quando si tratta di Istituti clericali si prevede che possano avere una chiesa (cfr. can. 282; can. 284 $\S 1$; can. 415 §1; can. 480 CCEO). L'autorità interna dell'Istituto deve

\footnotetext{
${ }^{46}$ Per esempio per aprire una nuova casa della Congregazione delle Suore Ancelle dell'Immacolata Concezione (di diritto pontificio) tale permesso lo dà la Superiora generale della Congregazione dopo aver ottenuto il parere del proprio consiglio generale - cfr. Costituzione delle Suore Ancelle dell'Immacolata Concezione, 132, p. 71.

${ }^{47}$ Nelle Chiese Orientali la benedizione del Vescovo significa anche il permesso di fare qualcosa.
} 
ricordare che quando si cambia il carattere dell'apostolato della casa si deve ricevere una nuova autorizzazione dal Vescovo eparchiale (cfr. 437; can. 509 §2 CCEO).

Sorge una domanda: è necessario chiedere il permesso dal Vescovo eparchiale quando si intende aprire una nuova casa però soltanto per due membri? La risposta è ovvia perché l'esistenza di una casa religiosa in una eparchia/esarcato ha un carattere ecclesiologico sia per la vita della stessa eparchia/esarcato che anche per l'Istituto.

Quando si tratta di aprire una prima casa sono necessari:

- la decisione della autorità competente dell'Ordine o della Congregazione;

- il permesso scritto del Vescovo eparchiale;

- il permesso scritto del Patriarca, se si tratta dell'erezione della prima casa dell'Istituto di diritto patriarcale in una eparchia/esarcato dentro al territorio della Chiesa patriarcale.

Invece se un Istituto, di qualsiasi condizione giuridica, vuole aprire una prima casa in un nuovo territorio, deve ottenere il permesso scritto dalla Sede Apostolica (cfr. can. 509 CCEO).

5.6. Soppressione della casa (cfr. can. 507; can. 510 CCEO)

Per sopprimere una casa dell'Ordine o della Congregazione si richiede:

- la decisione dell'autorità competente dell'Ordine o della Congregazione;

- il parere del Vescovo eparchiale. Questo requisito, dato da parte del Supremo Legislatore per gli Ordini o le Congregazioni, significa che per la validità dell'atto giuridico con il quale viene chiusa la casa, si richiede di sentire il parere del Vescovo eparchiale e non del Gerarca del luogo. Nel caso di inosservanza delle prescrizioni l'atto giuridico è nullo (cfr. can. $934 \S 2,2$ CCEO). Comunque anche se il parere del Vescovo eparchiale è contrario a quello dell'Ordine o della Congregazione, l'autorità competente dell'Istituto non è obbligata a seguirlo. 
Circa la soppressione dell'unica casa dell'Ordine o della Congregazione:

- se la casa appartiene ad un Ordine di diritto pontificio o patriarcale, il diritto di soppressione appartiene esclusivamente alla Sede Apostolica (cfr. can. 507 §1 CCEO);

- se la casa appartiene a una Congregazione di diritto pontificio o patriarcale, il diritto di soppressione di tale casa appartiene:

1) nel caso in cui si trovi fuori del territorio della Chiesa patriarcale è competente la Sede Apostolica;

2) nel caso in cui si trovi dentro del territorio della Chiesa patriarcale (cfr. can. $507 \S 2$; can. 934 §1 CCEO) è competente il Patriarca però sotto condizione che egli prima ascolti le persone interessate (che saranno i membri dell'Istituto e Vescovo eparchiale), e ottenga anche il consenso del Sinodo Permanente e della Sede Apostolica.

\section{Conclusione}

Le questioni di diritto consacrato raccolte nel nuovo Codice comune per le Chiese Cattoliche Orientali scelte e trattate in questo breve studio, ad un primo sguardo possono dare una presentazione generale, d'altra parte hanno una continuità logica.

Inoltre è necessario dire che esse sono state oggetto di domande poste durante i seminari formativi per i vari Istituti di vita consacrata della Chiesa Greco-Cattolica Ucraina.

I Monasteri sui iuris, gli Ordini e le Congregazioni di questa Chiesa sui iuris in tempi recenti attraversano non soltanto diverse difficoltà circa la propria riorganizzazione interna, ma si trovano anche nell'incertezza di trovare il proprio ruolo nella vita di ciascuna eparchia/esarcato. Come primo passo per aiutarli a trovare le giuste risposte per suddette situazioni, ma anche per indicare loro la giusta via nel loro cammino nella Chiesa, si richiede non soltanto di conoscere la presenza dei canoni circa l'organizzazione della vita consacrata nel Codice orientale, ma anche la retta interpretazione di essi.

Spesso questi Istituti mescolano la terminologia e i concetti giuridici circa la vita consacrata in Oriente cristiano e come conseguenza 
di questo applicano nella propria vita norme canoniche non corrispondenti alla condizione giuridica del proprio Istituto. Per esempio spesso si può trovare che i religiosi e le religiose appartenenti ad un Ordine o ad una Congregazione si denominino come monaci. Poi questo fatto viene trasmesso nella redazione dei loro Statuti dove sono citati dei canoni corrispondenti ai Monasteri. Dunque partendo dalla sbagliata terminologia giuridica si arriva alla scorretta redazione delle proprie norme particolari.

Inoltre spesso vengono notate alcune difficoltà circa la collaborazione fra gli Istituti di vita consacrata e il Vescovo eparchiale a livello eparchiale o a livello parrocchiale. Per esempio, essi seguendo la propria caratteristica si trovano ogni tanto nella difficoltà di aprire una nuova casa religiosa dentro il territorio della Chiesa patriarcale/arcivescovile maggiore.

Al termine di questo breve studio su alcuni aspetti del diritto consacrato nella nuova legislazione orientale si deve costatare il fatto che il Supremo Legislatore, tramite le diverse norme giuridiche, che a volte sembrano molto complicate, vuole raggiungere lo scopo principale di organizzare l'attività pastorale dentro l'eparchia/esarcato, con l'aiuto degli Istituti di vita consacrata.

\section{Some questions regarding the consecrated life in the oriental canonical legislation}

Title XII of the Codex Canonum Ecclesiarum Orientalium dedicated to the religious institutes in the oriental catholic traditions is made up of four chapters. The first chapter begins with some general preliminary canons (Art. I, cc. 410-432) on monks and other religious. These norms are to be born in mind while dealing specifically with monasteries, orders and congregations. Canonical questions regarding monasteries as such are treated in the second article (cc. 433-503), while the third refers to orders and congregations. Another important aspect of the oriental law on religious institutes is the typology of the forms of the consecrated life. This is quite different from the Latin legislation. In fact, the CCEO identifies five institutional and three individual kinds of the consecrated life, while the CIC simply distinguishes between religious and secular institutes. 